\title{
CARACTERIZAÇÃO DE MÉTODO LABORATORIAL ESPECÍFICO PARA VOLUMOSOS TRATADOS COM ÁLCALIS
}

\author{
CHARACTERIZATION OF A SPECIFIC LABORATORIAL METHOD \\ FOR ALKALI-TREATED ROUGHAGES
}

\author{
Maria de Lourdes Santorio Ciocca ${ }^{1}$ Marson Bruck Warpechowski $^{2}$ Eduardo Amato Bernhard ${ }^{3}$
}

\section{RESUMO}

O método específico para volumosos tratados com álcalis, descrito por LAU \& VAN SOEST (1981), foi avaliado, com palha de trigo, para caracterizar seus atributos, utilizandose a digestibilidade in vitro (DIVMO) como método referência. As estimativas de compostos fenólicos dissociados pelo tratamento com álcali (ABS-REF), de grupos tituláveis (GT) e de lignina residual associada à parede celular de materiais tratados (ABS-TIT) apresentaram, nesta ordem, repetibilidade decrescente. A precisão das medidas de GT e de ABS-TIT é mais baixa para palha tratada ( $\mathrm{NaOH} 4 \%$ ) em relação à não tratada, e resultados obtidos em diferentes corridas laboratoriais podem diferir significativamente. As estimativas da exatidão das medidas de ABSREF e GT, dada pela correlação entre seus valores e os de DIVMO obtidos com palha tratada com $\mathrm{O}$ a $4 \%$ de $\mathrm{NaOH}$, foram altas, $r_{A B S \text { REF }}=0,96(P<0,01)$ e $r_{G T}=-0,83(P<0,01)$, sendo reduzidas quando analisada em conjunto, palha tratada com uréia. Os resultados de ABS-TIT apresentaram alta imprecisão e não foram correlacionados com a DIVMO. Medidas de GT podem ser usadas para a seleção de palhas a serem submetidas a tratamentos com álcalis, e junto com medidas de ABS-REF, para estimar a digestibilidade de palhas tratadas.

Palavras-chave: tratamentos com álcali, palha de trigo, avaliação laboratorial.

\section{SUMMARY}

The specific method for alkali-treated roughages described by LAU \& VAN SOEST (1981) was evaluated, using wheat straw and in vitro digestibility (DIVMO) as reference method, aiming the characterization of its attributes. The estimated phenolic compounds dissociated during the alkali treat- ment (ABS-REF), titrable groups (GT) and residual lignin that remained in the cell wall after chemical treatment (ABS-TIT) showed, in this sequence, decreasing repeatability. The precision of the GT and ABS-TIT measurements with treated straw ( $\mathrm{NaOH}$ $4 \%$ ) were lower than with untreated straw, and results obtained in different batches can exhibit significant differences. The accuracy estimates of ABS-REF and GT measurements, obtained by correlation analysis between the values of 0 to $4 \% \mathrm{NaOH}$ treated straw and DIVMO, were high, $r_{A B S-R F F}=0.96(P<0.01)$ and $r_{G T}=$ $-0.83(P<0.01)$, and were reduced when the values obtained with urea treated straw was introduced in the analysis. The ABS-TIT values showed high imprecision and no significant relationship was found between them and DIVMO. GT measurements can be used to screen straws before alkali-treatments and, together with $A B S-R E F$ measurements, to predict straw-treated digestibility.

Key words: alkali-treatments, wheat straw, laboratorial evaluation.

\section{INTRODUÇÃO}

Vários métodos químicos foram desenvolvidos para aumentar a digestibilidade de volumosos de baixa qualidade para utilizá-los na alimentação de ruminantes. Para palhas, tratamentos com álcalis foram os primeiros descritos (JACKSON, 1977) e os empregados atualmente, excluem a remoção do agente químico residual por lavagem, evitando perda de matéria seca do material tratado (WILSON \& PIDGEN,1964). Em conseqüência deste procedimento, produtos resultantes das reações provocadas pelo álcali sobre a estrutura da parede

\footnotetext{
${ }^{1}$ Professora Adjunto, Doutora, Departamento de Zootecnia, Universidade Federal do Rio Grande do Sul. Faculdade de Agronomia. Universidade Federal do Rio Grande do Sul (UFRGS), Av. Bento Gonçalves, 7712, 90540-000 Porto Alegre, RS. E-mail: MLOURDES@admrgs.ufrgs.brAutor para correspondência.
}

'Professor Assistente, Mestre, Departamento de Zootecnia, Universidade Federal do Paraná.

${ }^{3}$ Estudante de Medicina Veterinária, UFRGS, Bolsista de Iniciação Científica, FAPERGS. 
celular permanecem no material tratado, e seus teores refletem-se na composição química. Esta, em termos de celulose, hemicelulose e lignina, apresenta poucas alterações em relação ao material inicial. Este fato torna-se especialmente importante em relação à lignina, que dissociada do complexo lignocelulósico por efeito do tratamento alcalino não afeta a digestibilidade, mas é detectada indistintamente da forma associada pelos métodos laboratoriais usuais (REXEN \& VESTERGAARD THOMPSEN, 1976). Portanto, o teor de lignina ou de frações que a contenham, determinados usualmente em alimentos $\mathrm{e}$ relacionados à digestibilidade de palhas e a de outros volumosos, não podem ser associados da mesma forma à qualidade de volumosos tratados. Para estabelecer adequada relação entre a composição química e valor nutritivo destes, métodos laboratoriais específicos são requeridos.

LAU \& VAN SOEST (1981) desenvolveram um método específico para avaliar, originalmente, a eficiência da delignificação de palhas de gramíneas submetidas a tratamentos alcalinos, que permite estimar sua digestibilidade. Estes autores fundamentaram o método laboratorial no pressuposto de que para gramíneas, que possuem grande número de ligações ésteres (lábeis a álcalis) entre compostos fenólicos e outros componentes da parede celular, a digestibilidade pode ser estimada a partir da quantidade de grupos saponificáveis medida em condições alcalinas e de uma determinação de compostos fenólicos associados a sua indigestibilidade, que sendo solúveis em álcali, fornecem uma base quantitativa para esta avaliação. Para estimar estes parâmetros, são preparadas fibras em detergente neutro de amostras de volumosos tratados e, em alíquotas do detergente, é determinada a absorbance em $280 \mathrm{~nm}$, estimando as unidades fenólicas dissociadas do complexo lignocelulósico pelo tratamento aplicado; subsequientemente, a fibra é tratada, à ebulição, com excesso de hidróxido de sódio padronizado, e o álcali não usado é titulado ácido (titulação de retorno), fornecendo um valor de saponificação do resíduo (determinação de grupos tituláveis); a absorbance em ultravioleta é medida também no extrato obtido após a saponificação, dando uma estimativa dos compostos fenólicos que permaneceram na parede do material tratado quimicamente, não solubilisados no processo.

Este método tem vantagens sobre outros aplicáveis e utilizados para volumosos tratados, como a determinação da digestibilidade in vitro em dois estágios (ALEXANDER, 1969) e espectroscopia de reflectância no infravermelho próximo (Russel et al. 1988, apud VAN SOEST, 1994), especialmente por utilizar procedimentos químicos comuns e por não exigir equipamentos específicos ou sofisticados. Também por essas razões, parece ser possível a utilização deste método por laboratórios brasileiros de nutrição animal, dada as indicações dos recursos neles encontrados presentemente (BELLAVER, 1993).

A adoção de novos métodos laboratoriais requer a caracterização de alguns de seus atributos em condições locais, uma vez que diferentes laboratórios não são capazes de manter todas as condições de operação num mesmo nível, especialmente no utilizado para o desenvolvimento do método. Manutenção do laboratório e de equipamentos e nível de treinamento de analistas são fontes comuns de variação entre laboratórios (WERNIMONT, 1985). De acordo com as recomendações dadas pela ICH (1995) para a validação de métodos químicos, parte das quais são de ampla aplicação, os atributos que devem ser caracterizados são os que permitem assegurar a exeqüibilidade do novo método por diferentes laboratórios, alcançando resultados apropriados.

Os objetivos deste trabalho foram caracterizar a precisão e a exatidão do método específico para palhas tratadas com álcalis, descrito por LAU \& VAN SOEST (1981), em condições comuns vigentes em laboratórios de nutrição animal locais, e testar a confiabilidade de resultados para avaliar esses materiais.

\section{MATERIAIS E MÉTODOS}

Esta avaliação foi realizada no Laboratório de Nutrição Animal do Departamento de Zootecnia da Universidade Federal do Rio Grande do Sul, em comparação com a digestibilidade in vitro da matéria orgânica ( DIVMO), adotada como método referência. Em todos os experimentos foi utilizada palha de trigo da variedade BR 23, coletada em um único local. Todo o material foi picado em picador de forragem equipado com peneira, e após a homogenização, foram retiradas 50 amostras de $100 \mathrm{~g}$, que foram armazenadas em sacos plásticos até o momento da aplicação dos tratamentos. Utilizou-se hidróxido de sódio comercial em concentrações de 1, 2, 3 e $4 \mathrm{~g}$ de $\mathrm{NaOH} / 100 \mathrm{~g}$ de matéria seca (MS). Para tratamentos com uréia a concentração foi de $6,2 \mathrm{~g} / 100 \mathrm{~g} \mathrm{MS}$, utilizando-se uréia comercial e reagente p.a. em duas formas de aplicação, líquida e incorporação das fontes por mistura a seco. Os tratamentos com $\mathrm{NaOH}$ e dois com uréia em solução foram feitos por aspersão de soluções, calculadas para fornecer a quantidade de água suficiente para elevar a umidade a $60 \%$. As amostras empregadas sem tratamento e as tratadas a seco foram aspergidas 
com água para que contivessem o mesmo nível de umidade. As amostras empregadas em cada experimento foram tratadas no mesmo dia e, após, mantidas em sacos plásticos duplos por, no mínimo, 72 horas para os tratamentos com $\mathrm{NaOH}$ e 28 dias, para os com uréia.

No Experimento I, foram utilizadas uma amostra de palha não tratada (PNT) e uma tratada com $4 \%$ de $\mathrm{NaOH}$, repetindo-se 20 vezes cada uma das três determinações do método para cada uma delas. As análises foram efetuadas em duas corridas laboratoriais com dez repetições por corrida para PNT, e em três para $\mathrm{NaOH} 4 \%$, com oito, oito e quatro repetições por corrida. Foi utilizado o delineamento experimental completamente casualizado com tratamentos constituídos pelas corridas laboratoriais.

No Experimento II, foram comparadas palhas tratadas com 1, 2 e $3 \%$ de $\mathrm{NaOH}$. Para a execução das três medidas preconizadas por LAU \& VAN SOEST (1981) foi adotado o delineamento experimental de blocos casualizados, constituídos por corridas laboratoriais executadas em dias diferentes. Foram utilizados 3 blocos com duas repetições de cada tratamento por bloco.

Como medida adicional, foi executado o Experimento III, em que foram utilizadas 6 amostras de cada um dos seguintes tratamentos: PNT, uréia comercial aplicada em solução (U-sol), uréia comercial cristalina, misturada a seco com a palha (U-crist), uréia p.a. aplicada em solução (U-pa) e $\mathrm{NaOH} 4 \%$. Foi analisada a regressão dos valores de DIVMO para os resultados da medida de grupos tituláveis e para os de absorbance $(280 \mathrm{~nm})$ determinada no detergente neutro utilizado para a obtenção das fibras.

Os resultados referentes à precisão foram expressos em desvios padrões e/ou coeficientes de variação. Todos os outros foram submetidos a análises da variância e de regressão, e as médias comparadas pelo teste de Tukey.

Os teores de matéria seca e de matéria orgânica das amostras foram determinados de acordo com as técnicas recomendadas pelo AOAC (1975) e os de fibra em detergente neutro, fibra em detergente ácido e lignina por GOERING \& VAN SOEST (1970). A digestibilidade in vitro foi determinada conforme procedimento de ALEXANDER (1969), em uma única corrida, em que foi incluída uma amostra de alfafa como padrão. A composição química inicial da palha de trigo utili- zada apresentou 90,63\% de matéria seca, e em base seca, $95,12 \%$ de matéria orgânica, $84,17 \%$ de fibra em detergente neutro, $56,56 \%$ de fibra em detergente ácido e 8,29\% de lignina.

\section{RESULTADOS E DISCUSSÃO}

Os resultados do Experimento I, tendo sido obtidos com alíquotas de mesma amostra tratada ou não tratada, preparadas independentemente, permitiram analisar a precisão de cada uma das medidas preconizadas no método avaliado. Esta análise foi feita em termos de repetibilidade e de precisão intermediária.

A repetibilidade, avaliada pela variação dentro de cada corrida (Tabela 1), foi decrescente na ordem: medida da absorbance do detergente neutro após o refluxo para a obtenção da fibra (ABS-REF) $>$ medida de grupos tituláveis (GT) > medida da absorbance do sobrenadante resultante após a titulação de retorno (ABS-TIT). Os coeficientes de variação observados para ABS-REF variaram entre 4,1 ( $\mathrm{NaOH} 4 \%, 3^{\mathrm{a}}$ corrida) e $6,6 \%$ (PNT, $2^{\mathrm{a}}$ corrida), para GT, entre 4,5 (PNT, $2^{a}$ corrida) e $16,8 \%(\mathrm{NaOH}$ $4 \%, 1^{\mathrm{a}}$ corrida) e para ABS-TIT, de 9,0 (PNT, $2^{\mathrm{a}}$ corrida) a $17,6 \%\left(\mathrm{NaOH} 4 \%, 1^{\mathrm{a}}\right.$ corrida). Esta ordem descrescente foi, como poderia ser esperado, inver-

Tabela 1 - Precisão das medidas de compostos fenólicos dissociados do complexo lignocelulósico pelo tratamento com álcali (ABSREF), do número de grupos tituláveis (GT) e da quantidade de lignina intacta associada à fibra (ABS-TIT) - Experimento I.

\begin{tabular}{|c|c|c|c|c|c|c|}
\hline $\begin{array}{c}\text { Tratamentos/ } \\
\text { Medidas }\end{array}$ & Corrida 1 & Corrida 2 & Corrida 3 & $\begin{array}{c}\text { Média } \\
\text { geral }\end{array}$ & $\mathrm{CV}(\%)$ & $P<t$ \\
\hline \multicolumn{7}{|l|}{ PNT } \\
\hline ABS-REF & 5,145 & 5,372 & $\ldots$ & 5,259 & 6,40 & NS \\
\hline $\mathrm{CV}(\%)$ & 5,73 & 6,60 & & & & \\
\hline GT & 0,599 & 0,613 & $\ldots$ & 0,606 & 8,13 & NS \\
\hline $\mathrm{CV}(\%)$ & 10,88 & 4,52 & & & & \\
\hline ABS-TIT & 6,873 & 8,989 & $\ldots$ & 7,931 & 17,13 & $* *$ \\
\hline $\mathrm{CV}(\%)$ & 12,65 & 8,99 & & & & \\
\hline \multicolumn{7}{|l|}{$\mathrm{NaOH}$} \\
\hline ABS-REF & 11,534 & 10,384 & 12,079 & 11,183 & 7,77 & $* *$ \\
\hline $\mathrm{CV}(\%)$ & 5,21 & 4,87 & 4,09 & & & \\
\hline GT & 0,272 & 0,338 & 0,318 & 0,308 & 15,03 & ** \\
\hline $\mathrm{CV}(\%)$ & 12,85 & 9,17 & 5,96 & & & \\
\hline ABS-TIT & 2,827 & 4,065 & 3,883 & 3,534 & 22,18 & $* *$ \\
\hline CV $(\%)$ & 17,55 & 14,46 & 13,20 & & & \\
\hline
\end{tabular}

ABS-REF: unidade de absorbance em $280 \mathrm{~nm} / \mathrm{mg}$ peso seco/ml de detergente após refluxo; GT: $\mathrm{mEq} \mathrm{HCl}$ g MS ( número de saponificação); $\mathrm{ABS}$-TIT: unidades de absorbance em $280 \mathrm{~nm} / \mathrm{mg}$ peso $\mathrm{seco} / \mathrm{ml}$ de sobrenadante após titulação.

NS: não significativo; $* * P<0,01$. 
samente relacionada com o número de operações envolvidas em cada uma das determinações. Também, como pode ser observado na Tabela 1, com a amostra tratada, os dados de GT e de ABS-TIT tiveram menor precisão que os da amostra de PNT. Estas observações foram atribuídas à modificação da fibra tratada, sobre a qual é feita a saponificação e ao número de operações necessárias para medida de ABS-TIT. A aplicação de hidróxido de sódio sobre a parede celular da palha de cereais, além da clivagem de ligações ésteres no complexo lignocelulósico, que pode liberar ácidos fenólicos, promove outras alterações, que incluem a desacetilação com perda de ésteres de ácido acético e solubilização de polissacarídios da matriz (CHESSON, 1981), com conseqüente alteração das interações entre as macromoléculas. Os resultados dessas reações, ausentes nos sistema de PNT, explicam, em parte, a diferença entre os resultados. Por outro lado, ainda que a composição das soluções obtidas com palha tratada e não tratada, usadas para as medidas finais de espectrofotometria sejam, conforme as indicações de CHESSON (1981), diferentes, os coeficientes de variação para ABS-REF para as duas amostra foram semelhantes e, portanto, a variabilidade dos dados de ABS-TIT pode ser atribuída à manipulação do extrato da amostra até o final da determinação.

A variação entre corridas, por abranger também a variação dentro de corridas, pode expressar a variação intralaboratorial total esperada. Quando na organização das corridas laboratoriais são incluídas fontes de variação cujos efeitos podem ser discernidos, os resultados desta precisão intermediária podem ser utilizados para identificar os fatores que têm maior contribuição para a variabilidade dos resultados finais (WERNIMONT,1985). No presente experimento, as principais fontes foram dias, partidas de reagentes e analistas que se alternaram na execução das operações. A redução da maior parte dos coeficientes de variação que ocorreu da $1^{\text {" para a }}$ $3^{\mathrm{a}}$ corrida foi atribuída ao treinamento alcançado pelos operadores, indicando que o treinamento pode melhorar consideravelmente a performance dos testes analíticos. Os valores dos coeficientes de variação obtidos com o total dos dados permaneceram na mesma ordem decrescente observada para a repetibilidade, e foram altos para as medidas de ABS-TIT.

De acordo com os resultados deste experimento, para empregar este método na avaliação de volumosos tratados com álcalis, todas as determinações devem ser concluídas em uma única corrida. Por outro lado, a determinação de grupos tituláveis ou saponificáveis preconizada neste método, quando adotada para palhas não tratadas representa outra aplicação potencial de interesse prático. Esta medida, obticla como parte de uma curva de titulação, possibilita estimar através do consumo de base padrão, a quantidade de álcali requerida para alcançar determinada digestibilidade. É bem documentado que palhas de cereais não respondem uniformemente a tratamentos com hidróxido de sódio e uréia e/ou amônia e parte desta variação tem sido atribuída a propriedades físico-químicas da parede celular destes materiais (VAN SOEST et al. 1984). Estes autores sugerem que a triagem de volumosos por técnicas laboratoriais relacionadas com a resposta ao tratamento químico poderia ser empregada, para assegurar maior eficiência dos mesmos. Segundo FAHEY et al. (1993) poderiam ser usadas para esta finalidade a determinação da capacidade de troca catiônica ou a saponificação como descrita por LAU $\&$ VAN SOEST (1981). A capacidade de troca catiônica de palha de trigo e de outros materiais, determinada nas mesmas condições em que foi realizado este experimento, apresenta precisão semelhante a observada neste trabalho para a medida de grupos tituláveis, coeficientes de variação de 4 a 13\%, e alta correlaçâo com a capacidade tamponante, $r=0,92$; $\mathrm{P}<0,01$ (WARPECHOWSKI \& CIOCCA, 1997). No entanto, o procedimento para a medida da saponificação apresenta as vantagens do uso de reagentes mais comuns, de ser menos laboriosa, podendo ser executada em menor intervalo de tempo. DIAS-DASILVA \& GUEDES (1990) demonstraram a possibilidade de classificar palhas de diversos cereais $(\mathrm{n}=24)$ quanto à suscetibilidade a tratamentos com uréia, encontrando alta correlação negativa entre resultados da determinação de grupos tituláveis e a resposta em digestibilidade in situ $(\mathrm{r}=-0,96$; $\mathrm{P}<0,01)$. Os resultados deste experimento sugerem que a utilização da medida de grupos tituláveis para esta seleção pode ser feita pela análise estatísitica de dados obtidos em dias diferentes como um único conjunto. uma vez que não houve diferença siginificativa entre corridas (Tabela 1).

Os resultados obtidos no Experimento II, para as três determinações preconizadas pelo método avaliado. encontram-se na Tabela 2. As variações dos valores numéricos encontradas foram congruentes com as indicações do trabalho original de LAU \& VAN SOEST (1981): com o aumento da força do tratamento com $\mathrm{NaOH}$, os valores de ABSREF tenderam a aumentar e os de GT e de ABSTIT, a diminuir. As respostas em termos de ABSREF e de GT foram lineares [ $r_{A B S-R E F}=0,81 ;(P<$ $\left.0,01) ; r_{\mathrm{GT}}=-0,76 ; \quad(\mathrm{P}<0,01)\right]$, mas a regressão dos valores de ABS-TIT para as quantidades de $\mathrm{NaOH}$ usadas nos tratamentos não foi significativa $(r=-0,35 ; P>0,10)$. 
Tabela 2 - Valores médios de ABS-REF, GT, ABS-TIT e DIVMO de amostras tratadas com 1,2 e $3 \%$ de $\mathrm{NaOH}$ - Experimento II.

\begin{tabular}{lcccc}
\hline Tratamentos & ABS-REF & GT & ABS-TIT & DIVMO \\
& & & & \\
\hline & & & & \\
$\mathrm{NaOH} \mathrm{1} \mathrm{\%}$ & $5,515 \mathrm{a}$ & $0,632 \mathrm{a}$ & $5,774 \mathrm{a}$ & $30,67 \mathrm{a}$ \\
$\mathrm{CV}(\%)$ & 6,35 & 12,00 & 46,02 & 7,01 \\
$\mathrm{NaOH} 2 \%$ & $6,838 \mathrm{ab}$ & $0,520 \mathrm{ab}$ & $5,372 \mathrm{a}$ & $41,08 \mathrm{~b}$ \\
$\mathrm{CV}(\%)$ & 11,67 & 16,22 & 30,51 & 4,04 \\
$\mathrm{NaOH} 3 \%$ & $7,973 \mathrm{~b}$ & $0,417 \mathrm{~b}$ & $4,211 \mathrm{a}$ & $49,68 \mathrm{c}$ \\
$\mathrm{CV}(\%)$ & 11,68 & 24,33 & 20,27 & 6,32 \\
& & & & \\
\hline
\end{tabular}

Médias na mesma coluna seguidas por letras distintas diferem pelo teste de Tukey $(\mathrm{P}<0,01)$.

ABS-REF: unidades de absorbance em $280 \mathrm{~nm} / \mathrm{mg}$ peso seco/ $\mathrm{ml}$ de detergente após refluxo; GT: $\mathrm{mEq} \mathrm{Hcl} \mathrm{/} \mathrm{g} \mathrm{MS} \mathrm{(} \mathrm{número}$ de saponificação); ABS-TIT: unidades de absorbantce em 280 $\mathrm{nm} / \mathrm{mg}$ peso seco/ ml de sobrenadante após titulação; DIVMO: digestibilidade in vitro da matéria orgânica (\%).

A dispersão dos dados experimentais deste experimento foi maior que a obtida no Experimento I (Tabela $1 \times$ Tabela 2 ). Como pode ser verificado com os dados da Tabela 2, os coeficientes de variação para ABS-REF ficaram entre 6 e $12 \%$, para GT entre 12 e 24\%, e os para ABS-TIT variaram entre 20 a $46 \%$. Este aumento era esperado uma vez que neste experimento, a variação total abrange a de todos procedimentos, incluindo a da amostragem do material original, a da aplicação do tratamento com álcali e a da preparação da amostra. Na condução deste experimento adotou-se a designação de operações/analista, eliminando assim uma fonte de variação dentro do laboratório. No entanto, como parte desta variabilidade observada com os resultados não compõe a precisão dos testes analíticos, é independente de qualquer controle laboratorial.

Não houve diferença significativa entre análises para os valores de ABSREF e de GT, mas os de ABS-TIT apresentaram diferença significativa (Tabela 3 ). Diante disso, para analisar a correlação entre o método avaliado e a DIVMO, foram agregados aos resultados deste experimento valores de ABS-REF e GT, obtidos com amostras de PNT e amostras tratadas com $4 \%$ de $\mathrm{NaOH}$ (6 repetições / tratamento), efetuando-se a análise com $n=30$ para estas duas determinações e com $n=18$ para ABS-TIT. Os valores médios de ABSREF, GT e DIVMO das amostras adicionais encontram-se na Tabela 4 . Os coeficientes de correlação obtidos foram 0,96 tivo.
$(\mathrm{P}<0,01)$ para ABS-REF, - 0,83 ( $\mathrm{P}<0,01)$ para GT e a relação entre estas duas variáveis e a DIVMO foi adequadamente descrita por regressões lineares: $\mathrm{Y}=$ 71,806 - 59,317. GT; $(\mathrm{P}<0,01)$ e $\mathrm{Y}=4,968+$ 4,916.ABS-REF ( $\mathrm{P}<0,01)$, onde $\mathrm{Y}=\mathrm{DIVMO}(\%)$. A correlação entre ABS-TIT e DIVMO não foi significativa $(r=0,33 ; \mathrm{P}>0,10)$.

A correlação entre os dois métodos analíticos fornece uma estimativa da exatidão daquele avaliado e, embora não exista um valor exato para fibras que permita uma avaliação específica deste atributo, a digestibilidade in vitro é amplamente aceita e usual em laboratórios de nutrição animal. Na descrição original, LAU \& VAN SOEST (1981) adotaram como referência a digestibilidade in vitro verdadeira da fibra em detergente neutro (GOERING \& VAN SOEST,1970), obtendo coeficientes de correlação mais altos, de 0,96-0,97 para absorbance após refluxo com detergente neutro, 0,95-0,99 para grupos tituláveis e 0,97-0,98 para absorbance após titulação. Os coeficientes de correlação entre ABS-REF e GT e a DIVMO encontrados neste trabalho podem ser considerados altos e o afastamento observado entre o valor do coeficiente obtido para GT e o da descrição original, pode ser atribuído a maior especificidade do padrão. No entanto, a ausência de associação entre ABS-TIT e DIVMO observada, contrariamente ao descrito por LAU \& VAN SOEST (1981), e entre esta medida e força do tratamento (Experimento I), indica que os procedimentos e condições adotados para o presente trabalho não foram suficientes para obter resultados aceitáveis para esta medida.

Os resultados obtidos no Experimento III encontram-se na Tabela 4. Como pode ser observado nesta tabela, a variação dos valores numéricos verificada para as duas medidas do método sugere um comportamento semelhante da palha submetida aos

Tabela 3 - Resumo da análise da variância das medidas de ABS-REF, GT e ABS-TIT - Experimento II.

\begin{tabular}{|c|c|c|c|c|}
\hline \multirow{2}{*}{$\begin{array}{c}\text { Fontes de } \\
\text { variação }\end{array}$} & \multirow[b]{2}{*}{ GL } & \multirow{2}{*}{$\frac{\text { ABS-REF }}{\text { Quadrado médio }}$} & \multirow{2}{*}{$\frac{\text { GT }}{\text { Quadrado médio }}$} & \multirow{2}{*}{$\begin{array}{c}\text { ABS-TIT } \\
\text { Quadrado médio }\end{array}$} \\
\hline & & & & \\
\hline$\%$ de $\mathrm{NaOH}$ & 2 & $9,0779 *$ & $0,0909 *$ & $3,8209 \mathrm{~ns}$ \\
\hline Corrida & 2 & $0,0458 \mathrm{~ns}$ & $0,0092 \mathrm{~ns}$ & $16,6693 *$ \\
\hline Erro & 13 & 0,6186 & 0,0074 & 1,4412 \\
\hline
\end{tabular}

$\mathrm{GL}=$ graus de liberdade; $*$ = significativo ao nível de $5 \%$; ns = não signific a- 
Tabela 4 - Valores médios de ABS-REF e de GT de palha não tratada (PNT) e de palha tratada com hidróxido de sódio ou uréia Experimento III.

\begin{tabular}{lcll}
\hline & ABS-REF & GT & DIVMO \\
\hline PNT & $5,8109 \pm 0,3871 \mathrm{~b}$ & $0,7569 \pm 0,0747 \mathrm{a}$ & $23,8430 \pm 1,6574 \mathrm{~d}$ \\
U-sol & $5,8267 \pm 0,3085 \mathrm{~b}$ & $0,4957 \pm 0,0609 \mathrm{~b}$ & $33,1177 \pm 5,4259 \mathrm{c}$ \\
U-p.a. & $5,8855 \pm 0,1660 \mathrm{~b}$ & $0,5371 \pm 0,0807 \mathrm{~b}$ & $38,4947 \pm 4,2605 \mathrm{ab}$ \\
U-crist & $5,9783 \pm 0,1951 \mathrm{~b}$ & $0,5261 \pm 0,0994 \mathrm{~b}$ & $40,3602 \pm 1,7323 \mathrm{~b}$ \\
NaOH $4 \%$ & $9,1409 \pm 2,2129 \mathrm{a}$ & $0,3927 \pm 0,0983 \mathrm{c}$ & $53,0040 \pm 4,7945 \mathrm{a}$ \\
\hline
\end{tabular}

Médias na mesma coluna seguidas por letras distintas diferem pelo teste de Tukey $(<0,05)$.

ABS-REF: unidades de absorbance em $280 \mathrm{~nm} / \mathrm{mg}$ peso seco/ml de detergente após refluxo; GT: mEq Hcl/g MS (número de saponificação).

diversos tratamentos. Na análise de regressão de DIVMO para os valores de ABS-REF e de GT, considerando-se juntos todos os tratamentos aplicados, as equações encontradas foram: para ABSREF, $\mathrm{Y}=10,778+4,134 \mathrm{x} ; \mathrm{r}=0,65(\mathrm{P}<0,01) \mathrm{e}$ para GT, $\mathrm{Y}=64,464-49,287 \mathrm{x} ; \mathrm{r}=-0,68(\mathrm{P}<0,01)$, onde $\mathrm{Y}=\operatorname{DIVMO}(\%)$. A ampla redução dos coeficientes de correlação para ABS-REF e para GT em relação aos observados no Experimento II, respectivamente, 0,96 e $-0,83$, sugere que a estimação da digestibilidade de palhas tratadas deve ser feita através de equações específicas por tratamento químico.

A execução das três medidas preconizadas neste método requer a organização de análises laboratoriais, dado o tempo necessário para completá-las para cada amostra.. Nas condições em que foi realizado este trabalho, com dois analistas, foram feitas as três medidas para doze materiais, ou seja, foram analisadas seis unidades experimentais em duplicata, em 6 a 8 horas. O treinamento de laboratoristas não reduz o tempo necessário para as análises, mas pode aumentar a precisão das medidas, como indicado no Experimento I, aproximando-a da observada com a DIVMO nos Experimentos II e III. De acordo com os resultados deste trabalho, entre as três medidas descritas por LAU \& VAN SOEST (1981), a medida da absorbance do detergente neutro utilizado na obtenção da fibra e a determinação de grupos tituláveis podem ser usadas nas condições comuns de laboratórios de nutrição animal locais, para avaliar palhas tratadas com álcalis, e nestas mesmas condições, a determinação de grupos tituláveis, que reflete a capacidade tamponante da fibra e tem sensibilidade mais alta, pode ser empregada para a seleção de volumosos a serem tratados quimicamente.

Agradecemos à Loiva Ana Marin da Fonte, pesquisadora da FEPAGRO /RS e à zootecnista Simone de David Antonio, pela orientação, execução e conferência dos dados experimentais de digestibilidade in vitro que compuseram este trabalho,

\section{REFERÊNCIAS BIBLIOGRÁFICAS}

ALEXANDER, R.H. The establishment of a laboratory prodedure for the "in vitro"determination of the digestibility. West of Scotland Agricultural College. Escocia: Auchincruive, 1969. 27 p. Research Bulletin, 42.

ASSOCIATION OF OFFICIAL AGRICULTURAL CHEMISTS. Official Methods of analysis. $12 \mathrm{ed}$ Washington: AOAC. 1975. $1094 \mathrm{p}$.

BELLAVER, C. Infraestrutura e linhas de pesquisa existentes nas instituições oficiais. In: WORKSOHP SOBRE A SITUAÇÃO DA PESQUISA EM NUTRIÇÃO NO BRASIL, 1993. Campinas, SP. CBNA (Não publicado)

CHESSON, A. Effects of sodium hydroxide on cereal straw in relation to the enhanced degradation of structural polysaccharides by rumen microrganisms. Journal of the Science of Food and Agriculture, Sussex, v. 32, p. 745-758, 1981.

DIAS-DA-SILVA, A.A.; GUEDES, C.V.M. Variability in the nutritive value of straw cultivars of wheat, rye and triticale and response to urea treatment. Animal Feed Science and Technology, Amsterdan, v. 28, p. 79-89, 1990.

FAHEY JR, G.C.; BOURQUIN, L.D.; TITGEMEYER, E.C.; $\boldsymbol{e}$ al. Postharvest treatment of fibrous feedstuffs to improve their nutritive value. In: JUNG, H.G.; BUXTON, D.R.; HATFIELD, R.D.; RALPH, J. Forage cell wall structure and digestibility. Madison: Amercan Society of Agronomy, Inc. 1993. Ch. 26. p.715-766.

GOERING, H.K.; VAN SOEST, P.J. Forage fiber analysis. Washington: Agricultural Research Service, USDA, 1970. 20 p. Agriculture handbook, 379.

INTERNATIONAL CONFERENCE ON HARMONISATION. Draft Guideline on validation of analytical procedures: definitions and terminology, Federal Register v. 60, p. 11260 , March 1, 1995

JACKSON, M.G. Review article: the alkali treatment of straws. Animal Feed Science and Technology, Amsterdan, v. 2, p. $105-130,1977$

LAU, M.M.; VAN SOEST, P.J. Titrable groups and soluble phenolic compounds as indicators of the digestibility of chemically treated roughages. Animal Feed Sciece and Technology, Amsterdan, v. 6, p. 123-131, 1981.

REXEN,F; VESTERGAARD THOMPSEN, K. The effect on digestibility of a new technique for alkali treatment of straw. Animal Feed Sicence and Technology, Amsterdan, v.1, p. 73-83, 1976.

VAN SOEST, P.J. Nutritional ecology of the ruminant. $2^{\text {nd }}$ ed. Ithaca: Cornell University Press, 1994. 476p.

VAN SOEST, P,J.; MASCARENHAS FERREIRA, A.; HARTLEY, R.D. Chemical properties of fibre in relation to nutritive quality of ammonia-treated forages. Animal Feed 
Science and Technology, Amsterdan, v. 10, p. 155-164, 1984.

WARPECHOWSKI, M.B.; CIOCCA, M.L.S. Propriedades físico-químicas da fibra em detergente neutro de ingredientes isolados e misturados. In: XXXIV REUNIÃO ANUAL DA SOCIEDADE BRASILEIRA DE ZOOTECNIA, 1997. Juiz de Fora, MG. Anais... Juiz de Fora. SBZ, 1997, v. 4, p.187190.
WERNIMONT, G.T. Use of estatistics to develop and evaluate analytical methods. Arlington, VA: AOAC. 1985. $182 \mathrm{p}$.

WILSON, R.K.; PIDGEN, W.J. Effects of a sodium hydroxide treatment on the utilization of wheat straw and poplar wood by rumen microorganisms. Canandian Journal of Animal Science, Ottawa, v. 44, p. 122-123, 1964.

Ciência Rural, v. 28, n. 3, 1998. 